\title{
APRENDIZAJE DE COMPETENCIAS: UN ANÁLISIS EMPÍRICO EN LOS ESTUDIOS UNIVERSITARIOS DE TURISMO EN ESPAÑA
}

\section{Introducción: Los nuevos retos en la gestión de las empresas del sector turismo}

En los albores del siglo XXI, los recursos humanos constituyen un factor clave de competitividad en el sector Turismo, ante los retos que se presentan de cara al futuro. La competencia en precios, como estrategia tradicional de la empresa turística, no sólo no ha conseguido sus objetivos de maximización de beneficios, sino que se ha mostrado como una alternativa imposible e insostenible en el tiempo en un entorno como el actual, caracterizado por la alta competencia y rivalidad internacional, la irrupción de nuevos destinos turísticos, la globalización del sector y la importante influencia de factores, políticos, económicos, sociales, medioambientales y tecnológicos (Alonso Almeida et al., 2006).

Además, los hábitos de consumo han tenido importantes cambios en los últimos veinte años. Se ha experimentado una generalización de la búsqueda de tiempo libre, los viajes por ocio y el disfrute de las vacaciones cortas a lo largo de todo el año gracias a la popularización de determinados medios de transporte como el avión-impulsada por la eclosión de las compañías de bajo coste-, o de destinos turísticos como el Caribe o China. Estos factores han forzado al sector a buscar un equilibrio entre cuatro elementos fundamentales: el precio, la calidad y diferenciación de la oferta, la rentabilidad y la sostenibilidad. Para ello, sus principales retos se orientan hacia la mejora del servicio, la profesionalización de los recursos humanos, la introducción de metodologías de calidad y atención al cliente, la diversificación de la oferta, la garantía de calidad, la optimización de la administración y gestión, la adecuación de los precios al mercado 
competitivo y la planificación sostenible que integre aspectos económicos con el medio ambiente.

Ante esta situación, los recursos humanos de los que disponen las empresas y las instituciones relacionadas con el turismo se configurarán como la pieza clave de todo el proceso, a pesar de que se ha detectado falta de estrategias de desarrollo en el área de recursos humanos que cubran las necesidades específicas del sector (Riley et al., 2002; Szivas et al., 2003).

Por ello, es urgente que la formación universitaria proporcione los profesionales que se requieren, dotándoles de las habilidades necesarias para no sólo no perder competitividad, sino también para incrementar aún más el protagonismo del sector dentro de las economías nacionales.

En el mundo, muchas organizaciones profesionales han empezado a definir las competencias necesarias para cada perfil profesional. Europa no podía obviar esta exigencia, y el Espacio Europeo de Educación Superior (EEES) ha sido el detonante que ha puesto en marcha este proceso. A continuación, se explicará el concepto y los distintos tipos de competencias que espera conseguir el EEES en los estudios de Turismo. Posteriormente, se enunciará el proceso de aprendizaje necesario para el desarrollo de estas competencias y los resultados obtenidos en el estudio realizado. El aspecto novedoso de este trabajo es la medición específica de este aprendizaje por parte de los estudiantes de Turismo, haciendo uso de la autoevaluación del estudiante antes de la evaluación tradicional formal de los estudios.

\section{La importancia del desarrollo de competencias en el sector turismo}

En Europa, con la implantación del nuevo EEES, se pretende, entre otros objetivos, dar respuesta a las necesidades de formación de un estudiante que debe estar preparado para afrontar el escenario de 
competitividad laboral actual y futura. En este sentido, más que la enseñanza se potencia su aprendizaje autónomo, a través de, entre otros aspectos, la acción tutorial y orientadora integrada en la acción docente (Gairín et al., 2004).

Una competencia tiene que ver con una combinación integrada de conocimientos, habilidades, motivos y actitudes conducentes a un desempeño adecuado y oportuno en diversos contextos. La flexibilidad y la capacidad de adaptación resultan claves para el nuevo tipo de logro que busca el trabajo y la educación como desarrollo general, para que las personas hagan algo con lo que saben (Huerta et al., 2003; UNESCO, 2003).

La propuesta del modelo general del EEES se fundamenta en los conceptos y terminología ligados al Proyecto Tuning (2000), para el que las competencias significan lo que el capital humano es capaz de ejecutar y su preparación y suficiencia para tareas y actividades determinadas. Estas capacidades tienen un sentido dinámico como combinación de atributos que habilitan para el desempeño competente, el cual es expresión del producto final originado por el proceso formativo (conocimiento, saber hacer, actitudes) y de acumulación de experiencias, y que, en principio, dura toda la vida.

Como el nuevo modelo de aprendizaje se fundamenta en el significado de "competencias", se tratará de esclarecer un poco más su significado. Según Lasnier (2000), competencia es un saber hacer complejo, producto de la integración, movilización y adecuación de capacidades, habilidades y conocimientos que, usados en situaciones parecidas no generalizables, permiten la consecución de los objetivos. Así, una competencia, como saber hacer de cierta complejidad, está integrada por capacidades (las cuales exigen conocimientos procedimentales y condicionales) y éstas por habilidades necesarias para una ejecución compleja, siendo las habilidades saber hacer simple con origen en conocimientos declarativos disciplinares (Le Boterf, 2002). 
A juicio de De la Cruz (2005), las competencias son megaconocimiento procedimental y condicional que permiten movilizar las capacidades de manera estratégica, esto es, conocimientos que requieren de un "arte de ejecución".

Esta autora propone la siguiente definición de competencia adaptada al EEES: "saber hacer complejo que exige un conjunto de conocimientos, habilidades, actitudes, valores y virtudes que garantizan la bondad y eficiencia de un ejercicio profesional responsable y excelente". Por tanto, la competencia profesional es "un saber hacer complejo que exige el dominio de los conocimientos, destrezas, actitudes, valores y virtudes específicas de la profesión y el arte de la ejecución pertinente a la situación".

Basándonos en estos conceptos, podemos definir "competencia" como la capacidad de un sujeto para aplicar en el momento más oportuno e idóneo la estrategia o conocimiento más adecuado en la realización de actividades, en el marco de una cultura organizativa o sistema de valores.

Según el Proyecto Tuning (2000), las competencias se pueden resumir en genéricas o transversales y específicas. Las transversales son las que todo profesional debe poseer, independientemente de su formación y del ámbito en el que vaya a desempeñar su actividad profesional; las específicas, las que el profesional ejerce de acuerdo con su formación profesional.

De esta forma, el profesional que posee las competencias requeridas para el desarrollo de su trabajo despliega una ventaja competitiva frente a otros que no las poseen (Nath y Raheja, 2001).

\subsection{Desarrollo de competencias en el sector turismo en el} ámbito del espacio europeo de educación superior (EEES)

Tener una competencia no es sólo poseer un conocimiento específico, incluso no es suficiente con saber aplicar ese conocimiento y desarrollar una labor, significa también ser capaz de desenvolverse 
en un entorno de trabajo, bajo unas condiciones concretas, y tomar decisiones que pueden implicar múltiples aspectos, como priorizar tareas, organizar el trabajo, coordinarse con otros compañeros y, finalmente, aplicar los conocimientos y desarrollar la tarea. Además, el profesional competente debe decidir las soluciones más convenientes, no sólo técnicamente hablando sino que, también, aplicando criterios de ética profesional y de buen hacer. Es pues la integración de aspectos: conocimientos, destrezas y actitudes lo que conforma la competencia. Para lograrla en el ámbito académico es preciso incluir como objetivos preferentes las competencias transversales, que actualmente forman parte de cualquier perfil profesional.

Si nos centramos en el aprendizaje de las competencias aplicadas al sector Turismo, el "Libro Blanco del Grado en Turismo" (ANECA, 2004), además de ofrecer un marco conceptual de los estudios de Turismo y presentar un análisis de su situación actual, tanto en España como en Europa, profundizó en el estudio de sus perfiles profesionales y, por último, de las competencias que, idealmente, deberían poseer los profesionales del sector.

Dado que, como es obvio, no se requieren las mismas capacidades si se va a trabajar en un subsector o ámbito turístico o en otro, o si se va a ocupar un puesto u otro, los responsables de estos estudios en las universidades españolas que participaron en el estudio valoraron de forma distinta las habilidades transversales que debían poseer por cada uno de los siete subsectores considerados (alojamiento, restauración, intermediación, transporte y logística, planificación y gestión pública de destinos, productos y actividades turísticas, y formación, investigación y consultoría) y por cada puesto específico perteneciente a cada uno de estos subsectores.

La relación entre competencias transversales consideradas y resultados medios obtenidos para todos los puestos y subsectores considerados en dicho estudio se recogen en la tabla 1. 
Tabla 1. Competencias transversales de los profesionales del Turismo

\begin{tabular}{|l|c|}
\hline COMPETENCIAS TRANSVERSALES & VALOR MEDIO \\
\hline COMPETENCIAS INSTRUMENTALES & 3,58 \\
\hline capacidad de análisis y síntesis & 3,72 \\
\hline capacidad de organización y planificación & 3,65 \\
\hline capacidad de comunicarse de forma oral y escrita en lengua nativa & 3,55 \\
\hline conocimiento de una lengua extranjera & 3,49 \\
\hline conocimientos de informática aplicada & 3,89 \\
\hline capacidad de gestión de la información & 3,81 \\
\hline capacidad de resolver problemas & 3,39 \\
\hline capacidad de tomar decisiones & \\
\hline CAPACIDADES PERSONALES & 3,69 \\
\hline capacidad de trabajar en equipo & 3,60 \\
\hline capacidad de realizar trabajo en equipos interdisciplinares & 3,28 \\
\hline de trabajar en un contexto internacional & 3,58 \\
\hline de mantener relaciones interpersonales & 3,35 \\
\hline de reconocer la diversidad y la multiculturalidad & 3,61 \\
\hline de poseer un razonamiento crítico & 3,83 \\
\hline de mantener un compromiso ético & \\
\hline CAPACIDADES SISTÉMICAS & 3,50 \\
\hline capacidad de aprender de forma autónoma & 3,78 \\
\hline de adaptarse a nuevas situaciones & 3,42 \\
\hline de ser creativo & 3,40 \\
\hline de ser líder & 3,32 \\
\hline de conocer otras culturas y costumbres & 3,53 \\
\hline de poseer iniciativa y espíritu emprendedor & 3,87 \\
\hline de perseguir la calidad & \\
\hline de ser sensible ante los temas medioambientales & \\
\hline & \\
\hline
\end{tabular}

Fuente: ANECA (2004).

Como puede apreciarse, la mayor parte de las competencias consideradas fueron muy bien evaluadas por estos responsables -la escala tenía un rango del 1 a 4 para evaluar el nivel de competencia requerido, en la cual el valor 1 significaba "Ningún" nivel requerido para cada competencia y el 4 "Mucho" nivel requerido-, indicando que son bastante necesarias para el desarrollo de la actividad profesional turística. Entre ellas destacan las capacidades de gestión de la información, de perseguir la calidad, de mantener un compromiso ético y de resolver problemas, todas ellas con una valoración superior a 3,8 puntos. La importancia de mantener un comportamiento ético por parte de los profesionales del Turismo se configura en diversos 
estudios como una competencia necesaria del comportamiento deseable (Hultsman, 1995; Fennell and Malloy, 1999), no sólo según el concepto kantiano del deber, sino en el sentido de no realizar promesas que no se pueden cumplir (Ross, 1975).

Por otro lado, tras múltiples reuniones de trabajo, los responsables universitarios de los estudios de Turismo en España lograron acordar una lista de treinta y dos competencias específicas de estos profesionales, algunas de las cuales recogen conocimientos disciplinares (lo que deben saber) y otras conocimientos operativos (lo que deben saber hacer).

De la larga lista de estas competencias específicas, las seleccionadas por los responsables de los estudios, por los profesores que imparten docencia en dichos estudios, por los directivos del sector y por los propios graduados, así como las valoraciones medias de todos ellos para los subsectores y puestos profesionales considerados, se recogen en la tabla 2 .

Tabla 2. Competencias específicas de los profesionales del Turismo

\begin{tabular}{|l|c|c|c|c|}
\hline COMPETENCIAS ESPECÍFICAS & $\begin{array}{c}\text { OPINIÓN } \\
\text { RESPONSABLES }\end{array}$ & $\begin{array}{c}\text { OPINIÓN } \\
\text { PROFESORES }\end{array}$ & $\begin{array}{c}\text { OPINIÓN } \\
\text { DIRECTIVOS } \\
\text { SECTOR }\end{array}$ & $\begin{array}{c}\text { OPINIÓN } \\
\text { GRADUADOS }\end{array}$ \\
\hline $\begin{array}{l}\text { Comprender los principios del } \\
\text { turismo }\end{array}$ & 3,11 & 3,05 & 3,33 & 3,48 \\
\hline $\begin{array}{l}\text { Analizar la dimensión económica } \\
\text { del turismo }\end{array}$ & 2,55 & 2,68 & 3,12 & 3,36 \\
\hline $\begin{array}{l}\text { Comprender el carácter dinámico y } \\
\text { evolutivo del turismo }\end{array}$ & 2,66 & 2,99 & 3,28 & 3,39 \\
\hline $\begin{array}{l}\text { Conocer las principales estructuras } \\
\text { político-administrativas turísticas }\end{array}$ & 2,58 & 2,70 & 2,90 & 3,11 \\
\hline $\begin{array}{l}\text { Convertir un problema empírico en } \\
\text { un objeto de investigación }\end{array}$ & 2,86 & 2,44 & 2,97 & 3,02 \\
\hline $\begin{array}{l}\text { Tener una marcada orientación de } \\
\text { servicio al cliente }\end{array}$ & 3,25 & 3,29 & 3,72 & 3,52 \\
\hline $\begin{array}{l}\text { Reconocer los principales agentes } \\
\text { turísticos }\end{array}$ & 2,83 & 2,80 & 3,29 & 3,30 \\
\hline $\begin{array}{l}\text { Evaluar los potenciales turísticos y la } \\
\text { prospección de su explotación }\end{array}$ & 2,92 & 2,76 & 3,33 & 3,48 \\
\hline
\end{tabular}




\begin{tabular}{|c|c|c|c|c|}
\hline $\begin{array}{l}\text { Analizar la información económico- } \\
\text { patrimonial de las organizaciones } \\
\text { turísticas }\end{array}$ & 2,70 & 2,46 & 2,70 & 2,89 \\
\hline Gestionar los recursos financieros & 2,43 & 2,53 & 3,22 & 3,03 \\
\hline $\begin{array}{l}\text { Definir objetivos, estrategias y } \\
\text { políticas comerciales }\end{array}$ & 2,83 & 2,83 & 3,47 & 3,39 \\
\hline $\begin{array}{l}\text { Dirigir y gestionar los distintos tipos } \\
\text { de entidades turísticas }\end{array}$ & 2,70 & 2,70 & 3,39 & 3,54 \\
\hline Manejar técnicas de comunicación & 3,04 & 3,08 & 3,43 & 3,39 \\
\hline $\begin{array}{l}\text { Comprender el marco legal que } \\
\text { regula las actividades turísticas }\end{array}$ & 2,68 & 2,86 & 3,10 & 3,22 \\
\hline $\begin{array}{l}\text { Trabajar en inglés como lengua } \\
\text { extranjera }\end{array}$ & 3,29 & 3,43 & 3,48 & 3,76 \\
\hline $\begin{array}{l}\text { Comunicarse de forma oral y escrita } \\
\text { en una segunda lengua extranjera }\end{array}$ & 2,67 & 2,95 & 3,39 & 3,63 \\
\hline $\begin{array}{l}\text { Comunicarse de forma oral y escrita } \\
\text { en una tercera lengua extranjera }\end{array}$ & 1,75 & 2,32 & 2,80 & 3,11 \\
\hline $\begin{array}{l}\text { Identificar y gestionar espacios y } \\
\text { destinos turísticos }\end{array}$ & 2,56 & 2,69 & 2,90 & 3,18 \\
\hline $\begin{array}{l}\text { Gestionar el territorio turístico } \\
\text { de acuerdo con los principios de } \\
\text { sostenibilidad }\end{array}$ & 2,20 & 2,38 & 2,99 & 3,47 \\
\hline $\begin{array}{l}\text { Conocer el procedimiento operativo } \\
\text { del ámbito del alojamiento }\end{array}$ & 2,12 & 2,47 & 3,29 & 3,34 \\
\hline $\begin{array}{l}\text { Conocer el procedimiento operativo } \\
\text { del ámbito de restauración }\end{array}$ & 2,42 & 2,25 & 3,15 & 3,09 \\
\hline $\begin{array}{l}\text { Conocer el procedimiento operativo } \\
\text { del ámbito de la intermediación }\end{array}$ & 2,58 & 2,57 & 3,06 & 3,28 \\
\hline $\begin{array}{l}\text { Analizar los impactos generados por } \\
\text { el turismo }\end{array}$ & 2,97 & 2,47 & 2,95 & 3,34 \\
\hline $\begin{array}{l}\text { Utilizar y analizar las TIC en los } \\
\text { distintos ámbitos del turismo }\end{array}$ & 3,35 & 3,08 & 3,44 & 3,35 \\
\hline $\begin{array}{l}\text { Comprender un plan público y las } \\
\text { oportunidades que se derivan para el } \\
\text { sector privado }\end{array}$ & 2,65 & 2,54 & 2,86 & 3,04 \\
\hline $\begin{array}{l}\text { Planificar y gestionar los recursos } \\
\text { humanos en las organizaciones } \\
\text { turísticas }\end{array}$ & 2,65 & 2,64 & 3,36 & 3,34 \\
\hline $\begin{array}{l}\text { Comprender el funcionamiento de } \\
\text { los destinos y estructuras turísticas } \\
\text { en el mundo }\end{array}$ & 2,62 & 2,59 & 2,67 & 3,05 \\
\hline $\begin{array}{l}\text { Conocer los objetivos, la estrategia } \\
\text { y los instrumentos públicos de } \\
\text { planificación }\end{array}$ & 2,44 & 2,69 & 2,67 & 3,10 \\
\hline
\end{tabular}


286 UN ANÁLISIS EMPÍRICO EN LOS ESTUDIOS UNIVERSITARIOS DE TURISMO EN ESPAÑA - José Miguel Rodríguez, Mar Alonso, Luis Rubio, Cristina Esteban

\begin{tabular}{|l|c|c|c|c|}
\hline $\begin{array}{l}\text { Trabajar en medios socioculturales } \\
\text { diferentes }\end{array}$ & 2,66 & 2,76 & 2,67 & 2,99 \\
\hline $\begin{array}{l}\text { Conocer las principales iniciativas } \\
\text { de puesta en valor del patrimonio } \\
\text { cultural }\end{array}$ & 2,31 & 2,49 & 2,66 & 3,21 \\
\hline $\begin{array}{l}\text { Comprender las características de la } \\
\text { gestión del patrimonio cultural }\end{array}$ & 1,98 & 2,29 & 2,59 & 3,06 \\
\hline $\begin{array}{l}\text { Detectar necesidades de } \\
\text { planificación técnica de } \\
\text { infraestructuras e instalaciones }\end{array}$ & 2,99 & 2,39 & 3,01 & 3,28 \\
\hline
\end{tabular}

Fuente: ANECA (2004).

Es interesante constatar las considerables discrepancias entre los cuatro colectivos encuestados. En principio, y en valores medios, los profesores universitarios piensan que son necesarios niveles más bajos de competencias para ejercer una actividad profesional en el ámbito del Turismo (2,67 sobre 4 puntos). Próximos a ellos se encuentran los responsables académicos de las titulaciones de Turismo $(2,69)$. En cambio, son bastante más exigentes los actuales directivos del sector $(3,10)$ y mucho más los egresados $(3,27)$, quienes piensan que se les demandará más en su futura profesión.

Por otro lado, aunque desde el punto de vista de los cuatro colectivos la capacidad de trabajar en inglés como lengua extranjera es la más importante (profesores: 3,43 y para graduados: 3,76 ) y la segunda para los otros dos colectivos (3,29 para los responsables de los estudios de turismo y 3,48 para los profesionales del sector), también destacan otras tres capacidades que son muy bien valoradas pero de forma distinta por dichos grupos. Los responsables de los estudios de Turismo opinan que la competencia más importante es ser capaz de utilizar las Tecnologías de Información y Comunicación (TIC) en el ámbito de trabajo $(3,35)$, los profesionales del sector, en cambio, eligen una marcada orientación al cliente $(3,72)$-competencia que es la segunda mejor valorada para los profesores de Turismo $(3,25)-$, mientras que los graduados piensan que la capacidad de comunicarse de forma oral y escrita en una segunda lengua extranjera es la segunda competencia que deseablemente deben desarrollar $(3,63)$. 
Estas opiniones enmarcan claramente los distintos puntos de vista de estos colectivos. Mientras que los estudiantes opinan que lo más importante son los idiomas, el resto de los grupos, aunque dan gran importancia a los conocimientos de inglés, los combinan con la orientación al cliente -es el caso de los profesionales del sector y de los profesores- o con la capacidad de utilizar las nuevas tecnologías de la información y las comunicaciones en su trabajo -esto ocurre con los responsables de los estudios de Turismo.

Ahora bien, para la adquisición de esas competencias es necesario que exista un adecuado proceso de aprendizaje. Los estudios realizados muestran que los estudiantes no poseen una estrategia de aprendizaje definida y, además, no todas las personas aprenden de la misma manera (Larkin 2002).

\subsection{El proceso de aprendizaje de competencias}

El EEES representa un nuevo paradigma en educación, al convertir la formación superior en una enseñanza-aprendizaje. Ello obliga implantar un nuevo modelo de aprendizaje basado en las competencias (Hernández Pina et al., 2005), caracterizado por:

a) Formación dirigida al desarrollo y evaluación de la competencia.

b) Énfasis en el logro de los resultados.

c) Enseñanza basada en el aprender haciendo y en el aprender a aprender.

d) Evaluación dirigida al conocimiento, las actitudes y el desempeño de la competencia como principal fuente de evidencia.

En la tabla 3 se muestran las directrices que el Centro Interamericano de Investigación y Documentación sobre Formación Profesional (CINTERFOR), dependiente de la Organización Internacional del Trabajo, elaboró para mostrar cómo debería ser la formación universitaria desde una perspectiva de las necesidades actuales de perfiles profesionales. 
288 UN ANÁLISIS EMPÍRICO EN LOS ESTUDIOS UNIVERSITARIOS DE TURISMO EN ESPAÑA - José Miguel Rodríguez, Mar Alonso, Luis Rubio, Cristina Esteban

Tabla 3. Orientaciones metodológicas sobre el nuevo modelo de formación

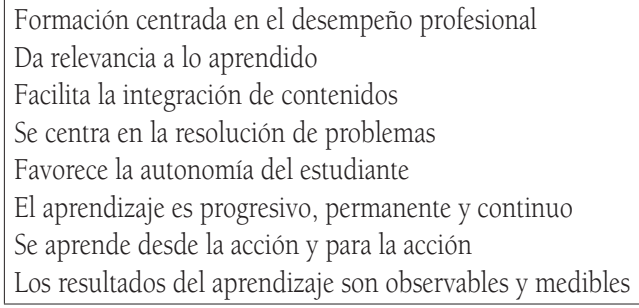

Fuente: CINTERFOR (2002).

Como se puede observar, hay un cambio radical en el modo de enseñar tradicional y en el rol del profesor. El estudiante se considera como un agente social, que persigue unas metas y es protagonista activo y constructivo de su aprendizaje, el que durará toda la vida. El profesor es un facilitador que guía, gestiona y conduce hacia un aprendizaje holístico y autónomo.

Ahora, el principal protagonista del nuevo escenario de educación es el alumno, en oposición a la educación tradicional basada en la enseñanza del profesor. Por ello, el EEES está también centrado en el alumno, y se fundamenta en la carga necesaria de trabajo que debe realizar para conseguir los objetivos proyectados. Éstos deben basarse en los resultados del aprendizaje de las competencias que el alumno debe adquirir, las cuales expresan su habilidad para desarrollar con éxito determinadas funciones. El estudiante deberá tomar un papel activo y participativo en su propia formación, de modo que se sienta más identificado con ella, y debe concienciarse de que su permanencia en la universidad, hasta la obtención del título correspondiente, es una etapa más dentro de su proceso de aprendizaje, que durará toda su vida. Esta actitud favorable hacia el aprendizaje, de manera participativa y con espíritu de superación ante las dificultades, le permitirá adquirir una predisposición para el autoaprendizaje y el trabajo continuo y organizado, lo que desembocará en la adquisición de una mayor competitividad.

Bajo estas premisas emanadas del EEES, se ha realizado un estudio con alumnos de Turismo. Se ha optado por conseguir que el 
estudiante siga un enfoque de aprendizaje profundo. Los estudiantes tienen, a priori, predilecciones o preferencias por uno u otro enfoque de aprendizaje, pero éstas pueden o no ser puestas en práctica, dependiendo del contexto (Biggs, 2005). Por eso, el profesor debe crear un entorno apropiado para este tipo de aprendizaje. Ciertos autores sugieren algunas características del contexto de aprendizaje asociadas con el enfoque de aprendizaje profundo (Ramsden, 2003; Biggs, 2005). En la tabla 4 se muestran las más relevantes utilizadas en el aula para esta investigación, junto con las acciones realizadas para llevarlas a cabo.

Tabla 4. Características del contexto de aprendizaje que fomenta el aprendizaje profundo

\begin{tabular}{|c|c|}
\hline CARACTERÍSTICAS DEL CONTEXTO & ACCIONES REALIZADAS \\
\hline $\begin{array}{l}\text { - Objetivos y expectativas claramente } \\
\text { definidas. }\end{array}$ & $\begin{array}{l}\text { - Desde el inicio en la guía de la diplomatura } \\
\text { se establecen los objetivos de la asignatura, } \\
\text { el temario, la planificación de clases y la } \\
\text { evaluación. }\end{array}$ \\
\hline $\begin{array}{l}\text { - Oportunidad para ejecutar una selección } \\
\text { responsable en el método y contenido de } \\
\text { estudio. }\end{array}$ & $\begin{array}{l}\text { - Se permite a los alumnos seleccionar un } \\
\text { tema entre los contenidos de la asignatura } \\
\text { y una empresa para hacer un trabajo } \\
\text { monográfico. }\end{array}$ \\
\hline $\begin{array}{l}\text { - Promover el interés y profundidad en los } \\
\text { contenidos de la materia. } \\
\text { - Enseñar a resolver problemas. }\end{array}$ & $\begin{array}{l}\text { - Se proporciona a los alumnos lecturas } \\
\text { complementarias. } \\
\text { - Por medio de la resolución y discusión de } \\
\text { casos prácticos. }\end{array}$ \\
\hline
\end{tabular}

Fuente: Elaboración propia a partir de Biggs (2005).

Para que el aprendizaje sea eficaz debe ser intencional, es decir, el estudiante se debe implicar activa y conscientemente en actividades cognitivas y metacognitivas dirigidas específicamente a pensar sobre algo y aprenderlo (Sinatra y Pintrich, 2003). El aprendizaje intencional es especialmente importante cuando el aprendiz necesita superar su conocimiento actual de un tema; esto es, cuando debe realizar un cambio conceptual (DiSessa et al., 2003; Sinatra y Pintrich, 2003). Este aprendizaje pone en marcha procesos esenciales que permiten revisar el conocimiento ya existente (Luque, 2003; Mason, 2003). El flujo del aprendizaje intencional es el siguiente: 
- Permite al estudiante atender a la nueva información de forma activa, pensar en dicha información y, por tanto, detectar las discrepancias con lo que ya sabía.

- Motiva al estudiante a dominar la materia, obligándolo a emplear un esfuerzo considerable para entenderla.

- Despliega una diversidad de estrategias de aprendizaje que le permiten revisar y cambiar su conocimiento acumulado de acuerdo con lo que escucha.

\section{Análisis empírico del proceso de aprendizaje de competencias en los estudios de turismo}

Partiendo de estas premisas y con el objetivo de analizar cómo se puede producir este pretendido proceso de aprendizaje y, por tanto, de adquisición de competencias, hemos elegido un grupo de estudiantes de primero de Turismo de la Universidad Autónoma de Madrid. Puesto que se trata de estudiantes de primer año no se ha valorado la experiencia profesional previa, prácticamente inexistente. Tampoco los estudios previos, pues son mayoritariamente de Bachillerato, y su estudio no proporcionaría datos relevantes para el objetivo propuesto. Este análisis se tendrá en cuenta en experiencias futuras con estudiantes de último año de estudios universitarios.

Se pesquisó cuáles eran tanto las competencias específicas y transversales que poseían los estudiantes, relacionadas con los estudios en cuestión, y cómo habían evolucionado desde el inicio hasta el final del curso, tras el oportuno proceso de aprendizaje.

Para ello, se diseñó un cuestionario estructurado de 27 preguntas, en escala de respuesta de Likert, de cinco categorías. Éste se aplicó a los estudiantes, que habitualmente asistían a clase, en dos ocasiones diferentes: el primer día de clase y el último del primer semestre del curso 2006-2007. La relación de los 14 ítems de la dimensión de competencias específicas fue fijada por los profesores que imparten dicha asignatura y la lista de los 13 ítems de la dimensión 
de competencias genéricas o transversales fue extraída de los veintitrés utilizados en el estudio anteriormente citado (ANECA, 2004), seleccionándose los que estaban más relacionados con el proceso de aprendizaje que iban a emprender en la asignatura Organización y Gestión de Empresas.

El perfil de los encuestados es el de un estudiante de Turismo, mayoritariamente mujer $(83,5 \%)$ y con una edad mediana al principio del estudio de 18 años. El instrumento de medida fue cumplimentado en la primera fase por 85 estudiantes y en la segunda por 82. Ellos debían evaluar su percepción sobre el nivel de competencia que tenían respecto de cada uno de los ítems al inicio y al final de curso.

Se seleccionó este método de evaluación porque es el que se utiliza habitualmente cuando se pretende que los alumnos tomen la responsabilidad de controlar y hacer juicios sobre aspectos de su propio aprendizaje (Boud, 1995). En este sentido, la autoevaluación es una forma de implicar al estudiante en su propio proceso formativo, ya que fomenta la reflexión y la autocrítica sobre éste. La autoevaluación, como tal, se recomienda como estrategia de aprendizaje y como instrumento de responsabilidad del propio alumno sobre su propio proceso, desde el cual el discente aprende a valorar, criticar y reflexionar sobre su propio trabajo (Calatayud, 1999; Anderson et al., 1994; Nunziati, 1990; Harris y Bell, 1986). Es un modo de educar en la responsabilidad y de conseguir que el estudiante progrese en su madurez y consiga un equilibrio personal y una integración social, tal como trata de potenciar el nuevo paradigma impulsado por el EEES.

La autoevaluación puede desarrollarse en cualquier momento del proceso de aprendizaje, si bien tiene más sentido al inicio y al final del mismo. Es práctica habitual utilizarla al inicio del periodo docente como método para conocer el nivel de conocimientos previos que de la materia tiene un estudiante. En cambio, la evaluación final solamente se desarrolla cuando finaliza ese periodo docente (Bound, 1995). Esta es la forma utilizada en este estudio. 
Para analizar la consistencia interna de los ítems que componen cada una de las dimensiones consideradas (competencias específicas de organización y gestión de empresas y competencias transversales al comienzo y al final del semestre) se empleó el coeficiente alfa de Cronbach. Los resultados para el primer grupo de variables fueron de 0,949, al comienzo, y 0,845, al final; para el segundo grupo de ítems 0,849 , al comienzo, y 0,831, al final. Por tanto, los resultados señalan una alta consistencia interna en las cuatro situaciones consideradas; esto es, un alto grado de relación entre los ítems que componen cada una de las dos escalas consideradas en los dos instantes de tiempo.

A continuación, se realizó un análisis de las distribuciones de frecuencias de cada una de las preguntas. Tras el mismo -como puede constatarse en las tablas 5 y 6 , en las que se recogen los porcentajes de estudiantes que dieron las puntuaciones máximas de 4 y 5-, se observó un incremento muy considerable al final del semestre (F) respecto al principio del mismo (P) en los ítems del Al al A14, lo que da a entender un aumento claro en la valoración que otorgaron a todos los indicadores relacionados con las competencias específicas de organización y gestión de empresas. En cambio, no hay un comportamiento claro en las preguntas de las competencias transversales, con aumentos en B2; B3, B4; B5; B6; B10; B11; B12 y $\mathrm{B} 13$, y reducciones en $\mathrm{B} 1$; B7; B8 B9; pero, en la mayoría de los casos, de menos entidad que los observados en el primer grupo de variables.

Tabla 5. Porcentaje de encuestados que otorgaron puntuaciones máximas a sus competencias específicas

\begin{tabular}{|l|c|c|}
\hline Competencias & Tiempo & Porcentaje \\
\hline \multirow{2}{*}{ A1 Dominar la terminología propia de la materia } & $\mathrm{P}$ & 34,118 \\
\cline { 2 - 3 } & $\mathrm{F}$ & 78,049 \\
\hline A2 Comprender e interpretar los fundamentos básicos de la & $\mathrm{P}$ & 42,353 \\
\cline { 2 - 3 } organización y dirección de empresas & $\mathrm{F}$ & 71,951 \\
\hline $\begin{array}{l}\text { A3 Aplicar estos fundamentos básicos a los principales tipos de } \\
\text { empresas turísticas }\end{array}$ & $\mathrm{P}$ & 22,353 \\
\cline { 2 - 3 } & $\mathrm{F}$ & 51,220 \\
\hline $\begin{array}{l}\text { A4 Analizar los principales principios de diseño organizativo que se } \\
\text { dan en las empresas turísticas }\end{array}$ & $\mathrm{P}$ & 15,294 \\
\cline { 2 - 3 } & $\mathrm{F}$ & 65,854 \\
\hline
\end{tabular}




\begin{tabular}{|c|c|c|}
\hline \multirow{2}{*}{$\begin{array}{l}\text { A5 Determinar la influencia de los factores de contingencia en el } \\
\text { diseño de la estructura organizativa de las empresas turísticas }\end{array}$} & $\mathrm{P}$ & 38,824 \\
\hline & $\mathrm{F}$ & 53,659 \\
\hline \multirow{2}{*}{$\begin{array}{l}\text { A6 Participar en el diagnóstico y diseño de las estructuras } \\
\text { organizativas turísticas, aplicando las últimas tendencias en el ámbito } \\
\text { del diseño organizativo }\end{array}$} & $\mathrm{P}$ & 12,941 \\
\hline & F & 45,122 \\
\hline \multirow{2}{*}{$\begin{array}{l}\text { A7 Aplicar la dirección participativa por objetivos en las } \\
\text { organizaciones turísticas }\end{array}$} & $\mathrm{P}$ & 16,471 \\
\hline & $\mathrm{F}$ & 50,000 \\
\hline \multirow{2}{*}{$\begin{array}{l}\text { A8 Concienciarse de la responsabilidad social que deben asumir las } \\
\text { empresas e instituciones turísticas }\end{array}$} & $\mathrm{P}$ & 48,235 \\
\hline & $\mathrm{F}$ & 75,610 \\
\hline \multirow{2}{*}{$\begin{array}{l}\text { A9 Aplicar la dirección estratégica a la dirección de las empresas } \\
\text { turísticas }\end{array}$} & $\mathrm{P}$ & 21,176 \\
\hline & $\mathrm{F}$ & 53,659 \\
\hline \multirow{2}{*}{$\begin{array}{l}\text { A10 Fijar adecuadamente la misión y los objetivos de una empresa } \\
\text { turística }\end{array}$} & $\mathrm{P}$ & 32,941 \\
\hline & $\mathrm{F}$ & 70,732 \\
\hline \multirow{2}{*}{ Al1 Evaluar la influencia del entorno en las empresas turísticas } & $\mathrm{P}$ & 38,824 \\
\hline & $\mathrm{F}$ & 82,927 \\
\hline \multirow{2}{*}{ A12 Analizar la competitividad de una empresa turística } & $\mathrm{P}$ & 27,059 \\
\hline & $\mathrm{F}$ & 67,073 \\
\hline \multirow{2}{*}{$\begin{array}{l}\text { A13 Diagnosticar las empresas turísticas bajo una perspectiva } \\
\text { estratégica }\end{array}$} & $\mathrm{P}$ & 10,714 \\
\hline & $\mathrm{F}$ & 30,488 \\
\hline \multirow{2}{*}{ A14 Formular e implantar estrategias en las empresas turísticas } & $\mathrm{P}$ & 16,667 \\
\hline & $\mathrm{F}$ & 45,122 \\
\hline
\end{tabular}

Fuente: Elaboración propia.

Tabla 6. Porcentaje de encuestados que otorgaron puntuaciones máximas a sus competencias genéricas o transversales

\begin{tabular}{|c|c|c|}
\hline Competencias & Tiempo & Porcentaje \\
\hline \multirow{2}{*}{ B1 Trabajar en equipo } & $\mathrm{P}$ & 82,353 \\
\hline & $\mathrm{F}$ & 73,171 \\
\hline \multirow{2}{*}{$\begin{array}{l}\text { B2 Resolver problemas (en el ámbito de la dirección y organización } \\
\text { de empresas turísticas) }\end{array}$} & $\mathrm{P}$ & 28,235 \\
\hline & $\mathrm{F}$ & 53,659 \\
\hline \multirow{2}{*}{ B3 Planificar y organizar su propio trabajo } & $\mathrm{P}$ & 75,294 \\
\hline & $\mathrm{F}$ & 76,829 \\
\hline \multirow{2}{*}{$\begin{array}{l}\text { B4 Gestionar (buscar, clasificar, almacenar y utilizar) la información } \\
\text { disponible }\end{array}$} & $\mathrm{P}$ & 65,882 \\
\hline & $\mathrm{F}$ & 80,488 \\
\hline \multirow{2}{*}{ B5 Tomar decisiones consensuadas } & $\mathrm{P}$ & 60,000 \\
\hline & $\mathrm{F}$ & 70,732 \\
\hline \multirow{2}{*}{ B6 Tener capacidad de análisis y de síntesis } & $\mathrm{P}$ & 44,706 \\
\hline & $\mathrm{F}$ & 65,854 \\
\hline \multirow{2}{*}{$\begin{array}{l}\text { B7 Conocer y aceptar la diversidad y multiculturalidad como } \\
\text { elemento clave de la vida }\end{array}$} & $\mathrm{P}$ & 75,294 \\
\hline & $\mathrm{F}$ & 70,732 \\
\hline \multirow{2}{*}{ B8 Tener un comportamiento responsable y ético } & $\mathrm{P}$ & 88,235 \\
\hline & $\mathrm{F}$ & 86,585 \\
\hline
\end{tabular}


294 UN ANÁLISIS EMPÍRICO EN LOS ESTUDIOS UNIVERSITARIOS DE TURISMO EN ESPAÑA - José Miguel Rodríguez, Mar Alonso, Luis Rubio, Cristina Esteban

\begin{tabular}{|l|c|c|}
\hline \multirow{2}{*}{ B9 Participar en su propio proceso de aprendizaje } & P & 84,706 \\
\cline { 2 - 3 } & $\mathrm{F}$ & 78,049 \\
\hline \multirow{2}{*}{ B10 Ser creativo } & $\mathrm{P}$ & 51,765 \\
\cline { 2 - 3 } & $\mathrm{F}$ & 69,512 \\
\hline \multirow{2}{*}{ B11 Tener iniciativa y espíritu emprendedor } & $\mathrm{P}$ & 65,882 \\
\hline \multirow{2}{*}{ B12 Estar motivado por la calidad } & $\mathrm{F}$ & 69,512 \\
\hline \multirow{2}{*}{ B13 Ser sensible ante los temas medioambientales } & $\mathrm{P}$ & 72,941 \\
\cline { 2 - 3 } & $\mathrm{F}$ & 84,146 \\
\hline
\end{tabular}

Fuente: Elaboración propia.

Seguidamente, se analizaron las medias aritméticas de los 27 ítems en los dos momentos considerados. Como puede observarse en las tablas 7 y 8 , casi todas las medias aritméticas al finalizar el primer semestre (F) son mayores que las obtenidas al comienzo del mismo (P). Destacan las diferencias en los valores de las preguntas referidas a las competencias específicas de organización y gestión de empresas (Al a A14), que registran incrementos del orden de un punto sobre cinco; especialmente en las preguntas: A4 (Analizar los principales principios de diseño organizativo que se dan en las empresas turísticas), con un aumento de 1,286, y A5 (Determinar la influencia que los factores de contingencia tienen en el diseño de la estructura organizativa de las empresas turísticas), con un incremento de 1,244. Sin embargo, las cuestiones referidas a las competencias transversales tienen variaciones sensiblemente menores e, incluso, negativas, como en los casos de B1 (Trabajar en equipo); B3 (Planificar y organizar su propio trabajo) y B9 (Participar en su propio proceso de aprendizaje).

La dispersión es pequeña, tanto en términos absolutos (desviación típica) como en relativos (coeficiente de variación de Pearson), en todas las preguntas, al comienzo y al final del semestre, implicando una alta representatividad de las medias aritméticas. En cualquier caso, es destacable la reducción que sufren las desviaciones típicas en las preguntas de las competencias específicas de organización y gestión de empresas (Al a Al4), mostrando que en este apartado existe un mayor consenso de los entrevistados al finalizar el semestre. 
Pero, como ocurría en el análisis de las medias, en las cuestiones referidas a las competencias transversales la evolución no es la misma, pues hay ítems que sufren reducciones y otros aumentos.

Tabla 7. Medias y dispersiones de las competencias específica

\begin{tabular}{|c|c|c|c|c|}
\hline Competencias & Tiempo & $\begin{array}{c}\text { Media } \\
\text { aritmética }\end{array}$ & $\begin{array}{c}\text { Desviación } \\
\text { típica }\end{array}$ & $\begin{array}{l}\text { Coeficiente } \\
\text { de variación }\end{array}$ \\
\hline \multirow{2}{*}{$\begin{array}{l}\text { Al Dominar la terminología propia de la } \\
\text { materia }\end{array}$} & $\mathrm{P}$ & 3,035 & 0,837 & 0,276 \\
\hline & $\mathrm{F}$ & 3,854 & 0,569 & 0,148 \\
\hline \multirow{2}{*}{$\begin{array}{l}\text { A2 Comprender e interpretar los } \\
\text { fundamentos básicos de la organización y } \\
\text { dirección de empresas }\end{array}$} & $\mathrm{P}$ & 3,271 & 0,931 & 0,285 \\
\hline & $\mathrm{F}$ & 3,793 & 0,643 & 0,170 \\
\hline \multirow{2}{*}{$\begin{array}{l}\text { A3 Aplicar estos fundamentos básicos a los } \\
\text { principales tipos de empresas turísticas }\end{array}$} & $\mathrm{P}$ & 2,835 & 0,998 & 0,352 \\
\hline & $\mathrm{F}$ & 3,500 & 0,758 & 0,216 \\
\hline \multirow{2}{*}{$\begin{array}{l}\text { A4 Analizar los principales principios } \\
\text { de diseño organizativo que se dan en las } \\
\text { empresas turísticas }\end{array}$} & $\mathrm{P}$ & 2,494 & 0,996 & 0,399 \\
\hline & $\mathrm{F}$ & 3,780 & 0,754 & 0,199 \\
\hline \multirow{2}{*}{$\begin{array}{l}\text { A5 Determinar la influencia de los factores } \\
\text { de contingencia en el diseño de la estructura } \\
\text { organizativa de las empresas turísticas }\end{array}$} & $\mathrm{P}$ & 2,329 & 0,851 & 0,365 \\
\hline & F & 3,573 & 0,685 & 0,192 \\
\hline \multirow{2}{*}{$\begin{array}{l}\text { A6 Participar en el diagnóstico y diseño } \\
\text { de las estructuras organizativas turísticas, } \\
\text { aplicando las últimas tendencias en el } \\
\text { ámbito del diseño organizativo }\end{array}$} & $\mathrm{P}$ & 2,447 & 0,994 & 0,406 \\
\hline & $\mathrm{F}$ & 3,378 & 0,731 & 0,216 \\
\hline \multirow{2}{*}{$\begin{array}{l}\text { A7 Aplicar la dirección participativa por } \\
\text { objetivos en las organizaciones turísticas }\end{array}$} & $\mathrm{P}$ & 2,588 & 1,003 & 0,388 \\
\hline & $\mathrm{F}$ & 3,463 & 0,757 & 0,219 \\
\hline \multirow{2}{*}{$\begin{array}{l}\text { A8 Concienciarse de la responsabilidad } \\
\text { social que deben asumir las empresas e } \\
\text { instituciones turísticas }\end{array}$} & $\mathrm{P}$ & 3,365 & 0,884 & 0,263 \\
\hline & F & 4,037 & 0,838 & 0,208 \\
\hline \multirow{2}{*}{$\begin{array}{l}\text { A9 Aplicar la dirección estratégica a la } \\
\text { dirección de las empresas turísticas }\end{array}$} & $\mathrm{P}$ & 2,753 & 0,925 & 0,336 \\
\hline & $\mathrm{F}$ & 3,500 & 0,774 & 0,221 \\
\hline \multirow{2}{*}{$\begin{array}{l}\text { A10 Fijar adecuadamente la misión y los } \\
\text { objetivos de una empresa turística }\end{array}$} & $\mathrm{P}$ & 2,976 & 0,926 & 0,311 \\
\hline & $\mathrm{F}$ & 3,878 & 0,776 & 0,200 \\
\hline \multirow{2}{*}{$\begin{array}{l}\text { A11 Evaluar la influencia que el entorno } \\
\text { tiene en las empresas turísticas }\end{array}$} & $\mathrm{P}$ & 3,235 & 0,959 & 0,296 \\
\hline & $\mathrm{F}$ & 4,061 & 0,673 & 0,166 \\
\hline \multirow{2}{*}{$\begin{array}{l}\text { A12 Analizar la competitividad de una } \\
\text { empresa turística }\end{array}$} & $\mathrm{P}$ & 2,800 & 1,033 & 0,369 \\
\hline & $\mathrm{F}$ & 3,683 & 0,815 & 0,221 \\
\hline \multirow{2}{*}{$\begin{array}{l}\text { A13 Diagnosticar las empresas turísticas } \\
\text { bajo una perspectiva estratégica }\end{array}$} & $\mathrm{P}$ & 2,321 & 0,933 & 0,402 \\
\hline & $\mathrm{F}$ & 3,195 & 0,637 & 0,199 \\
\hline \multirow{2}{*}{$\begin{array}{l}\text { Al4 Formular e implantar estrategias en las } \\
\text { empresas turísticas }\end{array}$} & $\mathrm{P}$ & 2,500 & 1,070 & 0,428 \\
\hline & $\mathrm{F}$ & 3,378 & 0,811 & 0,240 \\
\hline
\end{tabular}

Fuente: Elaboración propia. 
296 UN ANÁLISIS EMPÍRICO EN LOS ESTUDIOS UNIVERSITARIOS DE TURISMO EN ESPAÑA - José Miguel Rodríguez, Mar Alonso, Luis Rubio, Cristina Esteban

Tabla 8. Medias y dispersiones de las competencias genéricas o transversales

\begin{tabular}{|c|c|c|c|c|}
\hline Competencias & Tiempo & $\begin{array}{c}\text { Media } \\
\text { aritmética }\end{array}$ & $\begin{array}{l}\text { Desviación } \\
\text { típica }\end{array}$ & $\begin{array}{l}\text { Coeficiente } \\
\text { de variación }\end{array}$ \\
\hline \multirow{2}{*}{ B1 Trabajar en equipo } & $\mathrm{P}$ & 4,047 & 0,706 & 0,174 \\
\hline & $\mathrm{F}$ & 3,988 & 0,793 & 0,199 \\
\hline \multirow{2}{*}{$\begin{array}{l}\text { B2 Resolver problemas (en el ámbito de } \\
\text { la dirección y organización de empresas } \\
\text { turísticas) }\end{array}$} & $\mathrm{P}$ & 3,071 & 0,910 & 0,296 \\
\hline & F & 3,561 & 0,668 & 0,188 \\
\hline \multirow{2}{*}{ B3 Planificar y organizar su propio trabajo } & $\mathrm{P}$ & 3,988 & 0,794 & 0,199 \\
\hline & $\mathrm{F}$ & 3,951 & 0,752 & 0,190 \\
\hline \multirow{2}{*}{$\begin{array}{l}\text { B4 Gestionar (buscar, clasificar, almacenar } \\
\text { y utilizar) la información disponible }\end{array}$} & $\mathrm{P}$ & 3,753 & 0,815 & 0,217 \\
\hline & F & 4,098 & 0,730 & 0,178 \\
\hline \multirow{2}{*}{ B5 Tomar decisiones consensuadas } & $\mathrm{P}$ & 3,624 & 0,756 & 0,209 \\
\hline & F & 3,768 & 0,790 & 0,210 \\
\hline \multirow{2}{*}{ B6 Tener capacidad de análisis y de síntesis } & $\mathrm{P}$ & 3,471 & 0,853 & 0,246 \\
\hline & F & 3,768 & 0,775 & 0,206 \\
\hline \multirow{2}{*}{$\begin{array}{l}\text { B7 Conocer y aceptar la diversidad y } \\
\text { multiculturalidad como elemento clave de } \\
\text { la vida }\end{array}$} & $\mathrm{P}$ & 3,988 & 0,906 & 0,227 \\
\hline & F & 4,024 & 0,846 & 0,210 \\
\hline \multirow{2}{*}{$\begin{array}{l}\text { B8 Tener un comportamiento responsable } \\
\text { y ético }\end{array}$} & $\mathrm{P}$ & 4,224 & 0,643 & 0,152 \\
\hline & $\mathrm{F}$ & 4,280 & 0,725 & 0,169 \\
\hline \multirow{2}{*}{$\begin{array}{l}\text { B9 Participar en su propio proceso de } \\
\text { aprendizaje }\end{array}$} & $\mathrm{P}$ & 4,141 & 0,657 & 0,159 \\
\hline & $\mathrm{F}$ & 4,037 & 0,728 & 0,180 \\
\hline \multirow{2}{*}{ B10 Ser creativo } & $\mathrm{P}$ & 3,694 & 0,887 & 0,240 \\
\hline & $\mathrm{F}$ & 3,817 & 0,904 & 0,237 \\
\hline \multirow{2}{*}{$\begin{array}{l}\text { B11 Tener iniciativa y espíritu } \\
\text { emprendedor }\end{array}$} & $\mathrm{P}$ & 3,882 & 0,865 & 0,223 \\
\hline & $\mathrm{F}$ & 3,927 & 0,858 & 0,218 \\
\hline \multirow{2}{*}{ B12 Estar motivado por la calidad } & $\mathrm{P}$ & 3,941 & 0,836 & 0,212 \\
\hline & $\mathrm{F}$ & 4,122 & 0,760 & 0,184 \\
\hline \multirow{2}{*}{$\begin{array}{l}\text { B13 Ser sensible ante los temas } \\
\text { medioambientales }\end{array}$} & $\mathrm{P}$ & 4,200 & 0,768 & 0,183 \\
\hline & $F$ & 4,220 & 0,770 & 0,183 \\
\hline
\end{tabular}

Fuente: Elaboración propia.

Por último, es interesante mencionar que se han producido algunos ligeros cambios en un supuesto doble ranking, elaborado sobre la base del nivel de competencias que los estudiantes afirmaban poseer en el comienzo y en el final del curso, debido al proceso de aprendizaje efectuado. Por ejemplo, entre las competencias específicas, "Concienciarse de la responsabilidad social que deben asumir las empresas e instituciones turísticas" ocupaba el primer lugar al principio y ha pasado a ser el segundo al final, en tanto que "Evaluar 
la influencia que el entorno tiene en las empresas turísticas" ha pasado del tercer al primer lugar. Entre las competencias transversales, "Tener un comportamiento responsable y ético" era la más poseída, tanto al principio como al final; "Ser sensible a los temas medioambientales" ocupaba el segundo lugar también en los dos momentos; no obstante, "Estar motivado por la calidad" mejoró sensiblemente, subiendo del séptimo al tercer lugar.

\section{Análisis de las diferencias de género en el aprendizaje}

Para concluir, se realizó un análisis de cómo habían respondido las mujeres y los hombres a los 27 ítems mencionados, antes y después, y si había diferencias significativas en las dos categorías del atributo señalado. Como puede observarse en las tablas 9 y 10 , que recogen los valores medios de mujeres y hombres para cada pregunta y el valor crítico de probabilidad de un ANOVA, considerando como factor el sexo, hay diferencias claras en cómo responden los dos grupos de género.

Tabla 9. Medias aritméticas competencias de mujeres y hombres al principio del estudio

\begin{tabular}{|l|c|c|c|}
\hline \multirow{2}{*}{ Competencias } & Sexo & Media & Valor crítico \\
\hline \multirow{2}{*}{$\begin{array}{l}\text { A2 Dominar la terminología propia de la materia } \\
\text { de la organización y dirección de empresas }\end{array}$} & mujer & 3,06 & 0,605 \\
\cline { 2 - 4 } & hombre & 2,93 & \\
\hline \multirow{2}{*}{$\begin{array}{l}\text { A3 Aplicar estos fundamentos básicos a los principales } \\
\text { tipos de empresas turísticas }\end{array}$} & mujer & 3,39 & 0,032 \\
\cline { 2 - 4 } & hombre & 2,79 & \\
\cline { 2 - 4 } $\begin{array}{l}\text { A4 Analizar los principales principios de diseño } \\
\text { organizativo que se dan en las empresas turísticas }\end{array}$ & hombre & 2,89 & 0,282 \\
\hline \multirow{2}{*}{$\begin{array}{l}\text { A5 Determinar la influencia de los factores de } \\
\text { contingencia en el diseño de la estructura organizativa de }\end{array}$} & hombre & 2,57 & 0,150 \\
\cline { 2 - 4 } las empresas turísticas & mujer & 2,40 & 0,113 \\
\hline \multirow{2}{*}{$\begin{array}{l}\text { A6 Participar en el diagnóstico y diseño de las } \\
\text { estructuras organizativas turísticas, aplicando las últimas } \\
\text { tendencias en el ámbito del diseño organizativo }\end{array}$} & hujer & 2,00 & \\
\cline { 2 - 4 } & hombre & 2,00 & \\
\hline A7 Aplicar la dirección participativa por objetivos en las & mujer & 2,67 & 0,065 \\
\cline { 2 - 4 } organizaciones turísticas & hombre & 2,07 & \\
\hline
\end{tabular}




\begin{tabular}{|c|c|c|c|}
\hline \multirow{2}{*}{$\begin{array}{l}\text { A8 Concienciarse de la responsabilidad social que deben } \\
\text { asumir las empresas e instituciones turísticas }\end{array}$} & mujer & 3,44 & 0,043 \\
\hline & hombre & 2,93 & \\
\hline \multirow{2}{*}{$\begin{array}{l}\text { A9 Aplicar la dirección estratégica a la dirección de las } \\
\text { empresas turísticas }\end{array}$} & mujer & 2,81 & 0,080 \\
\hline & hombre & 2,36 & \\
\hline \multirow{2}{*}{$\begin{array}{l}\text { A10 Fijar adecuadamente la misión y los objetivos de } \\
\text { una empresa turística }\end{array}$} & mujer & 3,01 & 0,248 \\
\hline & hombre & 2,71 & \\
\hline \multirow{2}{*}{$\begin{array}{l}\text { A11 Evaluar la influencia del entorno en las empresas } \\
\text { turísticas }\end{array}$} & mujer & 3,30 & 0,054 \\
\hline & hombre & 2,79 & \\
\hline \multirow{2}{*}{ A12 Analizar la competitividad de una empresa turística } & mujer & 2,87 & 0,041 \\
\hline & hombre & 2,29 & \\
\hline \multirow{2}{*}{$\begin{array}{l}\text { A13 Diagnosticar las empresas turísticas bajo una } \\
\text { perspectiva estratégica }\end{array}$} & mujer & 2,40 & 0,085 \\
\hline & hombre & 1,93 & \\
\hline \multirow{2}{*}{$\begin{array}{l}\text { A14 Formular e implantar estrategias en las empresas } \\
\text { turísticas }\end{array}$} & mujer & 2,60 & 0,055 \\
\hline & hombre & 2,00 & \\
\hline \multirow{2}{*}{ B1 Trabajar en equipo } & mujer & 4,14 & 0,005 \\
\hline & hombre & 3,57 & \\
\hline \multirow{2}{*}{$\begin{array}{l}\text { B2 Resolver problemas (en el ámbito de la dirección y } \\
\text { organización de empresas turísticas) }\end{array}$} & mujer & 3,14 & 0,109 \\
\hline & hombre & 2,71 & \\
\hline \multirow{2}{*}{ B3 Planificar y organizar su propio trabajo } & mujer & 4,09 & 0,031 \\
\hline & hombre & 3,57 & \\
\hline \multirow{2}{*}{$\begin{array}{l}\text { B4 Gestionar (buscar, clasificar, almacenar y utilizar) la } \\
\text { información disponible }\end{array}$} & mujer & 3,86 & 0,006 \\
\hline & hombre & 3,21 & \\
\hline \multirow{2}{*}{ B5 Tomar decisiones consensuadas } & mujer & 3,67 & 0,294 \\
\hline & hombre & 3,43 & \\
\hline \multirow{2}{*}{ B6 Tener capacidad de análisis y de síntesis } & mujer & 3,54 & 0,116 \\
\hline & hombre & 3,14 & \\
\hline \multirow{2}{*}{$\begin{array}{l}\text { B7 Conocer y aceptar la diversidad y multiculturalidad } \\
\text { como elemento clave de la vida }\end{array}$} & mujer & 4,00 & 0,789 \\
\hline & hombre & 3,93 & \\
\hline \multirow{2}{*}{ B8 Tener un comportamiento responsable y ético } & mujer & 4,24 & 0,611 \\
\hline & hombre & 4,14 & \\
\hline \multirow{2}{*}{ B9 Participar en su propio proceso de aprendizaje } & mujer & 4,19 & 0,187 \\
\hline & hombre & 3,93 & \\
\hline \multirow{2}{*}{ B10 Ser creativo } & mujer & 3,77 & 0,120 \\
\hline & hombre & 3,36 & \\
\hline \multirow{2}{*}{ B11 Tener iniciativa y espíritu emprendedor } & mujer & 3,96 & 0,070 \\
\hline & hombre & 3,50 & \\
\hline \multirow{2}{*}{ B12 Estar motivado por la calidad } & mujer & 4,03 & 0,030 \\
\hline & hombre & 3,50 & \\
\hline \multirow{2}{*}{ B13 Ser sensible ante los temas medioambientales } & mujer & 4,14 & 0,225 \\
\hline & hombre & 4,43 & \\
\hline
\end{tabular}

Fuente: Elaboración propia. 
Tabla 10. Medias aritméticas competencias de mujeres y hombres al final del estudio

\begin{tabular}{|c|c|c|c|}
\hline Competencias & Sexo & Media & Valor crítico \\
\hline \multirow{2}{*}{ Al Dominar la terminología propia de la materia } & mujer & 3,91 & 0,073 \\
\hline & hombre & 3,63 & \\
\hline \multirow{2}{*}{$\begin{array}{l}\text { A2 Comprender e interpretar los fundamentos básicos } \\
\text { de la organización y dirección de empresas }\end{array}$} & mujer & 3,82 & 0,469 \\
\hline & hombre & 3,69 & \\
\hline \multirow{2}{*}{$\begin{array}{l}\text { A3 Aplicar estos fundamentos básicos a los principales } \\
\text { tipos de empresas turísticas }\end{array}$} & mujer & 3,50 & 1,000 \\
\hline & hombre & 3,50 & \\
\hline \multirow{2}{*}{$\begin{array}{l}\text { A4 Analizar los principales principios de diseño } \\
\text { organizativo que se dan en las empresas turísticas }\end{array}$} & mujer & 3,83 & 0,199 \\
\hline & hombre & 3,56 & \\
\hline \multirow{2}{*}{$\begin{array}{l}\text { A5 Determinar la influencia de los factores de } \\
\text { contingencia en el diseño de la estructura organizativa de } \\
\text { las empresas turísticas }\end{array}$} & mujer & 3,61 & 0,381 \\
\hline & hombre & 3,44 & \\
\hline \multirow{2}{*}{$\begin{array}{l}\text { A6 Participar en el diagnóstico y diseño de las } \\
\text { estructuras organizativas turísticas, aplicando las últimas } \\
\text { tendencias en el ámbito del diseño organizativo }\end{array}$} & mujer & 3,41 & 0,438 \\
\hline & hombre & 3,25 & \\
\hline \multirow{2}{*}{$\begin{array}{l}\text { A7 Aplicar la dirección participativa por objetivos en las } \\
\text { organizaciones turísticas }\end{array}$} & mujer & 3,45 & 0,831 \\
\hline & hombre & 3,50 & \\
\hline \multirow{2}{*}{$\begin{array}{l}\text { A8 Concienciarse de la responsabilidad social que deben } \\
\text { asumir las empresas e instituciones turísticas }\end{array}$} & mujer & 4,11 & 0,128 \\
\hline & hombre & 3,75 & \\
\hline \multirow{2}{*}{$\begin{array}{l}\text { A9 Aplicar la dirección estratégica a la dirección de las } \\
\text { empresas turísticas }\end{array}$} & mujer & 3,61 & 0,011 \\
\hline & hombre & 3,06 & \\
\hline \multirow{2}{*}{$\begin{array}{l}\text { A10 Fijar adecuadamente la misión y los objetivos de } \\
\text { una empresa turística }\end{array}$} & mujer & 3,92 & 0,276 \\
\hline & hombre & 3,69 & \\
\hline \multirow{2}{*}{$\begin{array}{l}\text { Al1 Evaluar la influencia del entorno en las empresas } \\
\text { turísticas }\end{array}$} & mujer & 4,03 & 0,405 \\
\hline & hombre & 4,19 & \\
\hline \multirow{2}{*}{ A12 Analizar la competitividad de una empresa turística } & mujer & 3,74 & 0,181 \\
\hline & hombre & 3,44 & \\
\hline \multirow{2}{*}{$\begin{array}{l}\text { A13 Diagnosticar las empresas turísticas bajo una } \\
\text { perspectiva estratégica }\end{array}$} & mujer & 3,24 & 0,174 \\
\hline & hombre & 3,00 & \\
\hline \multirow{2}{*}{$\begin{array}{l}\text { A14 Formular e implantar estrategias en las empresas } \\
\text { turísticas }\end{array}$} & mujer & 3,41 & 0,485 \\
\hline & hombre & 3,25 & \\
\hline \multirow{2}{*}{ B1 Trabajar en equipo } & mujer & 4,05 & 0,183 \\
\hline & hombre & 3,75 & \\
\hline \multirow{2}{*}{$\begin{array}{l}\text { B2 Resolver problemas (en el ámbito de la dirección y } \\
\text { organización de empresas turísticas) }\end{array}$} & mujer & 3,61 & 0,217 \\
\hline & hombre & 3,38 & \\
\hline \multirow{2}{*}{ B3 Planificar y organizar su propio trabajo } & mujer & 4,03 & 0,053 \\
\hline & hombre & 3,63 & \\
\hline \multirow{2}{*}{$\begin{array}{l}\text { B4 Gestionar (buscar, clasificar, almacenar y utilizar) la } \\
\text { información disponible }\end{array}$} & mujer & 4,17 & 0,082 \\
\hline & hombre & 3,81 & \\
\hline \multirow{2}{*}{ B5 Tomar decisiones consensuadas } & mujer & 3,76 & 0,805 \\
\hline & hombre & 3,81 & \\
\hline \multirow{2}{*}{ B6 Tener capacidad de análisis y de síntesis } & mujer & 3,79 & 0,645 \\
\hline & hombre & 3,69 & \\
\hline
\end{tabular}


300 UN ANÁLISIS EMPÍRICO EN LOS ESTUDIOS UNIVERSITARIOS DE TURISMO EN ESPAÑA - José Miguel Rodríguez, Mar Alonso, Luis Rubio, Cristina Esteban

\begin{tabular}{|l|c|c|c|}
\hline \multirow{2}{*}{$\begin{array}{l}\text { B7 Conocer y aceptar la diversidad y multiculturalidad } \\
\text { como elemento clave de la vida }\end{array}$} & mujer & 4,08 & 0,267 \\
\cline { 2 - 4 } & hombre & 3,81 & \\
\hline \multirow{2}{*}{ B8 Tener un comportamiento responsable y ético } & mujer & 4,30 & 0,571 \\
\cline { 2 - 4 } & hombre & 4,19 & \\
\hline \multirow{2}{*}{ B9 Participar en su propio proceso de aprendizaje } & mujer & 4,09 & 0,171 \\
\cline { 2 - 4 } & hombre & 3,81 & \\
\hline \multirow{2}{*}{ B10 Ser creativo } & mujer & 3,82 & 0,982 \\
\hline \multirow{2}{*}{ B11 Tener iniciativa y espíritu emprendedor } & hombre & 3,81 & \\
\hline \multirow{2}{*}{ B12 Estar motivado por la calidad } & mujer & 3,97 & 0,361 \\
\cline { 2 - 4 } & hombre & 3,75 & \\
\hline \multirow{2}{*}{ B13 Ser sensible ante los temas medioambientales } & mujer & 4,14 & 0,730 \\
\cline { 2 - 4 } & hombre & 4,06 & \\
\cline { 2 - 4 } & mujer & 4,23 & 0,854 \\
\hline
\end{tabular}

Fuente: Elaboración propia.

En primer lugar, las mujeres dan una valoración media más alta en todas las preguntas formuladas al principio. El promedio de las medias aritméticas es de 2,85 para las mujeres y de 2,39 para los hombres en las competencias específicas (una diferencia absoluta de 0,46 puntos), y de 3,90 y 3,57 en las competencias transversales (una diferencia de 0,33 puntos a favor de las mujeres). Asimismo, se observaron discrepancias estadísticamente significativas en ocho competencias, aquellas que arrojaron un valor crítico de la probabilidad inferior o igual a 0,05 en el análisis de la varianza mencionado. De estas ocho, la mitad corresponde a cada grupo de competencias.

En segundo lugar, al final del semestre, aunque en general las mujeres siguen dando una valoración media más alta, las diferencias se reducen. En tres preguntas (A7 "Aplicar la dirección participativa por objetivos en las organizaciones turísticas"; A11 "Evaluar la influencia del entorno en las empresas turísticas" y B5 "Tomar decisiones consensuadas"), los hombres dieron una puntuación media superior, y tan sólo hay un ítem (A9 "Aplicar la dirección estratégica a la dirección de las empresas turísticas") que tiene un valor crítico inferior que 0,05 y que, por tanto, apunta a una diferencia estadísticamente significativa. Sin embargo, los promedios de las medias aritméticas siguen siendo mayores en las mujeres: 3,69 y 3,50 en las competencias específicas, 
esto es, una diferencia absoluta de 0,19 puntos -claramente inferior a los 0,46 puntos que había al principio- a favor de las mujeres, y de 4,00 en las mujeres y 3,82 en los hombres en las competencias transversales, es decir, una diferencia de 0,18, también inferior a los 0,33 puntos del principio.

Por lo tanto, las mujeres, en general, dan puntuaciones más altas, pero las diferencias se reducen en el proceso de aprendizaje tendiendo a igualar las valoraciones de los colectivos, lo cual es un síntoma de un adecuado proceso de aprendizaje que, en definitiva, logra elevar y equilibrar las competencias de los futuros profesionales del sector Turismo.

\section{Conclusiones}

No cabe duda que, ante los nuevos e importantes retos que debe afrontar el sector Turismo receptivo español, el factor humano es el recurso más importante con el que debe contar. En este sentido, el análisis de las competencias de estos profesionales se muestra como un elemento clave de cara al futuro. Las empresas necesitan buenos profesionales para prestar unos servicios con la calidad que demandan los turistas.

Por estas razones, tanto las competencias transversales como las específicas jugarán un destacado papel en la competitividad de empresas e instituciones y, para adquirirlas, un adecuado proceso de aprendizaje -basado en un enfoque "profundo" y ajustado a las directrices emanadas del EEES- se hace imprescindible, de cara a satisfacer las necesidades de unos clientes cada vez más experimentados, formados y exigentes.

Este estudio cuenta con una limitación, porque no se da opción a los estudiantes de evaluar la importancia de cada una de las competencias para su futuro profesional, debido a que las competencias que se evalúan son parte de una lista cerrada. Esta restricción se mitiga por el consenso logrado con los profesionales y 
los graduados del sector respecto de las competencias necesarias para un futuro profesional del turismo.

Del análisis de los datos se han obtenido unos resultados que permiten evidenciar un adecuado proceso de aprendizaje, más que aceptable en el grupo de las competencias transversales y muy intenso en el grupo de las competencias específicas propias de la asignatura elegida. Además, se aprecian algunos interesantes cambios en el orden del dominio de las competencias analizadas, tanto transversales como específicas.

Por otro lado, el estudio de las diferencias de género ha mostrado que, si bien al principio del semestre las mujeres se atribuían un nivel de competencias apreciablemente superior al de los hombres, tras el proceso de aprendizaje estas distancias se redujeron ostensiblemente, dejándose de apreciar diferencias significativas de género. En todo caso, en un futuro muy próximo se profundizará en esta línea de investigación. Adicionalmente, se pretende enriquecer estudios futuros sobre aprendizaje de competencias, añadiendo otras variables, como la autoevaluación de las competencias específicas y transversales para el ingreso del estudiante en el mundo laboral, la experiencia profesional previa, si existe, y los estudios previos.

El aprendizaje basado en competencias pone énfasis en que el futuro profesional del sector Turismo "sea capaz de valorar su proceso de enseñanza y aprendizaje y sea un aprendiz activo, autónomo, reflexivo, y responsable". Ello es coherente con la alta valoración que se da a la responsabilidad social de empresas e instituciones turísticas, en términos de un comportamiento responsable y ético y respeto por los temas medioambientales, actitudes fundamentales en el entorno dinámico mundial en el que se desenvuelve el sector Turismo.

\section{Referencias bibliográficas}

Alonso Almeida, M.; Barcos Redín L. y Martín Castilla J. I. (2006) Gestión de la calidad de los procesos turísticos. Madrid: Editorial Síntesis. 
Anderson, G.; Boud, D y Sampson, J. (1994) Expectations of quality in the use of learning contracts. The International Journal of Capability in Higher Education, 1(1), pp. 22-31.

ANECA (2004) Libro Blanco del Grado en Turismo. Madrid: Ministerio de Educación y Ciencia.

Biggs, J. (2005) Calidad del aprendizaje universitario. Madrid: Narcea.

Boud, D. (1995) Enhancing learning through self assessment. London: Kogan.

Calatayud Salom, A. (1999) La participación del alumno en el proceso evaluador. Revista Educadores, pp. 190-191.

CINTERFOR (2002) Informe 2002. (Sitio en Internet) Disponible en: hppt://www.cinterfor.org.uy.

De la Cruz, M. A. (2005) Taller sobre el proceso de aprendizaje-enseñanza de competencia. ICE, Universidad de Zaragoza.

DiSessa, A. A.; Elby A. y Hammer D. (2003) J’s epistemological stance and strategies. In Sinatra G. M. and Pintrich P. R. (eds.) Intentional conceptual change. Mahwah, NJ: Erlbaum.

Fennell, D. A. and Malloy D. C. (1999) Measuring the ethical nature of tourism operators. Annals of Tourism Research, 26(4): pp. 928-943.

Gairín, J.; Feixas, M.; Guillmón C. y Quinquer D. (2004) La tutoría académica en el escenario europeo de la Educación Superior. Revista Interuniversitaria de Formación del Profesorado, 18(1): pp. 61-77.

Harris, D y Bell, CH. (1986) Evaluating and assessing for learning. London: Kogan Page.

Hernández Pina, F; Martínez Clares, P. Da Fonseca Rosario P. y Rubio Espín M. (2005) Aprendizaje, competencias y rendimiento en Educación Superior. Madrid: La Muralla.

Huerta, J.; Pérez I. S. y Castellanos A. R. (2003) Desarrollo curricular por competencias profesionales integrales. (Sitio en Internet) Disponible en http://educacion.jalisco.gob.mx/consulta/educar /13/ 13Huerta.html

Hultsman, J. (1995) Just Tourism: An Ethical Framework. Annals of Tourism Research, 22: pp. 553-567.

Larkin, T. (2002) A Global Approach to learning styles. Ponencia presentada en el Congreso: "Frontiers in Education Conference". Boston, Massachusetts. 
304 UN ANÁLISIS EMPÍRICO EN LOS ESTUDIOS UNIVERSITARIOS DE TURISMO EN ESPAÑA - José Miguel Rodríguez, Mar Alonso, Luis Rubio, Cristina Esteban

Lasnier, F. (2000) Réussir la formation par compétences. Montréal: Guérin.

Le Boterf, G. (2002) Gestión de las competencias. Barcelona: Editorial Gestión.

Luque, M. L. (2003) The role of domains specific knowledge in intelectual conceptual change. En Sinatra, G. M. and Pintrich, P. R. (eds.) Intencional conceptual change. Mahwah, NJ: Erlbaum.

Mason, L. (2003) Personal epistemologies and intentional conceptual change. En Sinatra, G. M. and Pintrich, P. R. (eds.) Intentional conceptual change. Mahwah, NJ: Erlbaum.

Nath, R. and Raheja, R. (2001) Competencies in the Hospitality Industry. Journal of Service Research, 1(1): pp. 25-33.

Nunziati, G. (1990) Pour construire un dispositif d'evaluation formatrice. Cahiers pédagogiques, 280, pp. 47-64.

Ramsden, P. (2003) Learning to teach in higher education. Londres: Routledge.

Riley, M.; Ladkin A. and Szivas E. (2002) Tourism Employment: Analysis and Planning. Clevedon: Channel View.

Ross, W. D. (1975) The Right and the Good. En Struhl, K. J. and Struhl, P. R. (eds.) Ethics in Perspective. New York: Random House, pp. 100-107.

Sinatra, G. M. and Pintrich, P. R. (eds.) (2003) Intencional conceptual change. Mahwah, NJ: Erlbaum.

Szivas, E.; Riley M. and Airey D. (2003) Labor Mobility into Tourism: Attraction and Satisfaction. Annals of Tourism Research, 30: pp. 64-76.

TUNING (2000) Tuning Educational Structures in Europe. Informe final. Proyecto Piloto-Fase 1. Bilbao: Universidad de Deusto.

UNESCO (2003) Enseñanza y aprendizaje. (Sitio en Internet) Disponible en http://innovemos.unesco.cl/eya/index.act

Recibido: 12 de marzo de 2008

Aceptado: 19 de mayo de 2008 